\title{
Validation of a mixed-layer closure. I: Theoretical tests
}

\author{
Douglas K. Lilly ${ }^{\mathrm{a} *}$ and Bjorn Stevens ${ }^{\mathrm{b}}$ \\ ${ }^{\text {a }}$ University of Oklahoma, Norman, Oklahoma, USA \\ b University of California, Los Angeles, California, USA
}

\begin{abstract}
A flux closure formula introduced earlier for predicting entrainment into a mixed layer is tested through analytic solution of the kinetic energy equation and through large-eddy simulation. For this purpose, the closure formulation and the simulation model are applied to the 'smoke cloud' mixed layer, that includes both radiative cooling at the cloud top and surface heating or cooling at the surface, but without the effects of latent heat. The unique aspect of the closure, height weighting of the integral of buoyancy flux, is found to be directly related to the ratio of coefficients of the dissipation and diffusion terms of the energy equation.

The simulation results indicate that the previous expression for predicting the entrainment flux is about $10 \%$ too small. Results of analytic solutions of the energy equation and the simulations are in general agreement. Disagreements are believed to be due to approximations in the energy equation and possible imperfections in the simulation model.

A special issue investigated is the condition for decoupling of energy by surface radiative cooling. The maximum negative buoyancy flux before decoupling predicted by the flux closure and by the energy equation differ by almost a factor of 2, but it is not clear which is better. Copyright (c) 2008 Royal Meteorological Society
\end{abstract}

KEY WORDS entrainment; kinetic energy equation; radiative cooling; large-eddy simulation; smoke cloud; flux closure

Received 12 March 2007; Revised 12 October 2007; Accepted 17 October 2007

\section{Introduction and flux closure model}

The primary objective of this work and that described in part II is to test an earlier closure formula (Lilly, 2002b) for predicting entrainment into a mixed layer. The observational comparisons provided in II were actually completed first, but withheld from publication until the closure could be better verified theoretically, which is the burden of this paper. With aid of an analytic solution of the kinetic energy equation and comparison with the results of a large-eddy simulation model, we have perhaps obtained a deeper understanding of the closure and its variants. In this section, we introduce the closure and derive some of its solutions. In section 2, we describe results of large-eddy simulations and compare them with those of the closure model. In section 3 we work with the energy equation and show how its solutions relate to those of the closure equation and the simulations. Section 4 describes and comments on several apparent discrepancies of the simulations and/or theory. Section 5 reports the conclusions.

The closure, which we call a 'flux closure', is given as follows:

$$
B_{\mathrm{i}}=-A_{n} \int_{0}^{z \mathrm{i}} B\left(\frac{z}{z_{\mathrm{i}}}\right)^{n} \frac{\mathrm{d} z}{z_{\mathrm{i}}},
$$

where $B=\overline{w^{\prime} b^{\prime}}$ is the turbulent buoyancy flux, with $w^{\prime}$ and $b^{\prime}$ the deviations of vertical velocity and buoyancy

* Correspondence to: Douglas K. Lilly, $9774175^{\text {th }} \mathrm{Rd}$, Amherst, Nebraska 68812, USA. E-mail: dlilly66@aol.com $\left(g \theta^{\prime} / \theta\right)$ from the overbarred means, which may be horizontal and/or temporal. The height $z_{\mathrm{i}}$ is that of the mixed layer top, and $B_{\mathrm{i}}$ is the flux at that level. $A_{n}$ and $n$ are dimensionless quantities to be determined. $B$ is positive throughout most of the mixed layer, and the evidently negative value at its top supports entrainment into it, with the entrainment rate given as $B_{\mathrm{i}}$ divided by the buoyancy jump across the top of the mixed layer. Thus, (1.1) is regarded as an entrainment predictor. A more traditional expression does not have the $z / z_{\mathrm{i}}$ factor, that is $n=0$. A non-zero value, specifically $n=$ 1 , along with $A_{n} \approx 3$, allows accurate (by comparison to simulations and observations in II) predictions in conditions including both surface heating and radiative cooling, particularly in the presence of cloud cover in the mixed layer. The rationale is that positive $n$ takes account of dissipation between the layers of energy generation and removal, so that positive buoyancy flux near the mixed-layer top is more effective at producing energy there than the same amount of flux at lower levels.

The rationale suggested above is not instantly convincing, but our analysis in section 3 adds weight to it. The analysis is based on the 'smoke cloud' assumption (Lilly, 1968), in which the mixed layer is unsaturated, but a cloud of radiatively opaque material is present throughout. The smoke cloud incorporates the dynamic principles of mixed-layer entrainment without the thermodynamic complexities of a real cloud. Consideration 
of a dry boundary layer without radiative cooling does not allow unique predictions of $A$ and $n$.

The linear buoyancy flux profile for an idealized smoke cloud is

$$
\left.\begin{array}{c}
B+F=B_{0}\left\{1-\left(z / z_{\mathrm{i}}\right)\right\}+\left(B_{\mathrm{i}}+F_{\mathrm{i}}\right) z / z_{\mathrm{i}} \text { for } z \leq z_{\mathrm{i}} \\
B=0 \text { for } z>z_{\mathrm{i}}
\end{array}\right\},
$$

where $F$ is the net outgoing radiation flux. In the most accurate theories and simulation models, $F=F_{\mathrm{i}}$ at the mixed-layer top and drops exponentially to zero within the cloud layer. In most real and simulated boundary layer clouds, the layer of radiating material is quite thin, typically $1-5 \%$ of the mixed-layer depth. Here we assume that, as in the Lilly (1968) model, all the radiation is emitted from the mixed-layer top, rather than being distributed within the smoke cloud, so that $F=0$ except at and above $z_{\mathrm{i}}$. Then (1.2) is extended to

$$
\left.\begin{array}{l}
B=B_{0}\left\{1-\left(z / z_{\mathrm{i}}\right)\right\}+\left(B_{\mathrm{i}}+F_{\mathrm{i}}\right) z / z_{\mathrm{i}} \text { for } z<z_{\mathrm{i}} \\
B=B_{\mathrm{i}} \text { for } z=z_{\mathrm{i}} \\
B=0 \text { for } z>z_{\mathrm{i}}
\end{array}\right\}
$$

Thus, there is a double discontinuity at the mixed-layer top. $B=B_{\mathrm{i}}+F_{\mathrm{i}}$ (positive or negative) just beneath the top, then drops suddenly to $B_{\mathrm{i}}$ (negative) at the top, and rises to zero just above it. The lower discontinuity is due to the radiation flux assumption and the upper one to the assumption that entrainment occurs at exactly the mixed-layer top. This is an approximation also, since in all real mixed layers there is some shear across their tops, which leads to a finite transition layer in which the Richardson number is near a critical value. In addition, most analyses of observations and simulations use horizontal or temporal averages to establish the mean profiles. As shown by Lilly (2002a), such averaging smooths out discontinuities like those in (1.3), which only remain if averaging is done relative to the local mixed-layer top, as assumed here. With these caveats, the integral in (1.1) is evaluated as

$$
B_{\mathrm{i}}=-\frac{A_{n}}{n+2}\left(\frac{B_{0}}{n+1}+B_{\mathrm{i}}+F_{\mathrm{i}}\right) .
$$

We may solve for $B_{\mathrm{i}}$ to obtain

$$
B_{\mathrm{i}}=-\frac{A_{n}}{A_{n}+n+2}\left(\frac{B_{0}}{n+1}+F_{\mathrm{i}}\right) .
$$

This expression is a closure prediction for entrainment buoyancy flux, based on the assumption of a vanishing radiation depth in the cloud and the closure equation (1.1). On the basis of earlier simulations, Lilly (2002b) found that $n=1, A_{1}=3$ were approximately valid, consistent with the closure prediction

$$
B_{\mathrm{i}}=-B_{0} / 4-F_{\mathrm{i}} / 2 .
$$

After eliminating $B_{\mathrm{i}}$ from (1.3) we find an expression for the buoyancy flux profile that uses only the forcing quantities:

$$
\left.\begin{array}{l}
B=B_{0}+\left(-\frac{5 B_{0}}{4}+\frac{F_{i}}{2}\right) \frac{z}{z_{\mathrm{i}}} \text { for } z<z_{\mathrm{i}} \\
B=-\frac{B_{0}}{4}-\frac{F_{\mathrm{i}}}{2} \text { for } z=z_{\mathrm{i}} \\
B=0 \text { for } z>z_{\mathrm{i}}
\end{array}\right\} .
$$

Insertion of (1.7) into (1.1) again produces (1.6). For a real wet cloud, the flux profile is more complicated than (1.3) or (1.7). With use of (1.7), however, the entrainment flux predicted from (1.1) is found in II to agree well with observational results from DYCOMS2.

An interesting question that has not, to our knowledge, been much considered is the limit of negative buoyancy flux at the bottom of a mixed layer that is driven by cloud-top radiative cooling. The turbulent energy in such a layer can be sustained against a certain amount of negative surface flux, but beyond that the surface layer becomes decoupled from the turbulent driving, and the kinetic energy drops to nearly zero near the surface. The proper way of formulating the mixed-layer equations for this case is not completely clear, but we provide two possible solutions: one derived here and based on an extension of the flux closure, and the other based on an energy equation, presented in section 3 .

The assumed linear flux profile remains the same as (1.3) with $B_{0}$ negative, but how negative can it be? A proposed answer is to treat the lower boundary similarly to the upper, so that the hypothesized maximum negative surface buoyancy flux is a mirror image of the negative entrainment flux at the top, i.e.

$$
B_{0}=-A \int_{0}^{z \mathrm{i}} B\left(1-\frac{z}{z_{\mathrm{i}}}\right)^{n} \frac{\mathrm{d} z}{z_{\mathrm{i}}} .
$$

One might interpret this as a condition for entrainment of a marginally thin inversion layer underneath the mixed layer, which is maintained by surface cooling. After inserting the profile of the first of (1.3), the condition becomes a linear combination of $B_{0}, B_{\mathrm{i}}$ and $F_{\mathrm{i}}$. Application of (1.1) leads to another linear relation between the same variables, and combined solution yields $B_{\mathrm{i}} / F_{\mathrm{i}}$ and $B_{0} / F_{\mathrm{i}}$ as constants. For the coefficients applied in II, $n=1$ and $A_{1}=3$, the result is

$$
B_{0}=-\frac{2}{15} F_{\mathrm{i}}, \quad B_{\mathrm{i}}=-\frac{7}{15} F_{\mathrm{i}} .
$$

Thus, the negative surface buoyancy flux that leads to decoupling is predicted to be $2 / 15$ of the radiative flux, but the cloud-top flux is barely affected. The net effect on the mixed layer is cooling, but not even as much as the radiative flux generates, because the cloud-top entrainment warming is greater than the surface cooling.

From the large-eddy simulation results presented in section 3, we find that a slightly improved closure prediction, in place of (1.6), is

$$
B_{\mathrm{i}}=-0.274 B_{0}-0.548 F_{\mathrm{i}} .
$$


This leads to small changes in (1.7) and (1.9).

\section{Large-eddy simulation}

The smoke cloud model of the previous section was subjected to large-eddy numerical simulation, using a small array of forcing conditions. The algorithms used in the model are described by Stevens et al. (2005). In the threedimensional motion equations, the momentum advection is centred in time and fourth-order centred in space, while the scalar equations for potential temperature and smoke apply a slope-limited, forward-in-time upwind technique. The Smagorinsky-Lilly subgrid closure is used on both scalar and velocity variables. The grid mesh consists of 128 points in each horizontal dimension with a spacing of $25 \mathrm{~m}$. In the vertical, a stretched grid is applied with spacing $12.5 \mathrm{~m}$ near the surface, contracting to $5 \mathrm{~m}$ between 575 and $825 \mathrm{~m}$, and stretched above to a spacing of about $20 \mathrm{~m}$ at the top of the model, at $1500 \mathrm{~m}$. This yields a finer mesh and a larger domain than what was used in the original smoke cloud model of Bretherton et al. (1997). The model employs a variable time step to maintain a Courant number near 0.7, resulting in time steps of about $1 \mathrm{~s}$, depending on the motion field development. Simulations are performed for 4 hours of simulated time.

The initial potential temperature is $290 \mathrm{~K}$ in the lowest $700 \mathrm{~m}$, with a sharp inversion jump of $7 \mathrm{~K}$ and a weak stable layer above, with a potential temperature gradient $\approx 0.3 \mathrm{~K} \mathrm{~km}^{-1}$. The smoke density at the surface and through the mixed layer is initially dimensionless unity in the mixed layer and zero above. The smoke is dynamically and thermally inactive except to serve as a marker for the mixed-layer top. The simulations performed are labelled as 0030, 0060, 1545, 3000, 3030, $3060,4530,60 \mathrm{~m} 20,60 \mathrm{~m} 10,6000,6030$ and 6060 , all in $\mathrm{W} \mathrm{m}{ }^{-2}$, with $1 \mathrm{~W} \mathrm{~m}^{-2}$ equivalent, after dividing by the density and specific heat of air, to $\approx 0.0865 \mathrm{~K} \mathrm{~cm} \mathrm{~s}^{-1}$. In each run the first two numbers correspond to the cloudtop radiant cooling rate and the last two correspond to the surface heating rate. The $\mathrm{m} 10$ and $\mathrm{m} 20$ labels indicate surface cooling. The radiant flux is applied at the first grid point below the mixed-layer top, which is defined locally to be where the smoke density falls below 0.5 . Initially, there is no motion, but a small random temperature fluctuation, together with the surface heat flux, generates turbulence within the first 1500 seconds. After that, the mixed layer grows by entrainment a few tens of metres, and it warms or cools a fraction of a degree.

Table I shows results that are independent of or have been averaged over time. These include the mixed layer depth $z_{\mathrm{i}}$, entrainment (and mixed-layer growth) rate $w_{\mathrm{e}}$, the potential temperature jump at the interface $\Delta \theta$, and the deduced entrainment buoyancy flux

$$
B_{\mathrm{i}}=-g w_{\mathrm{e}} \Delta \theta / \bar{\theta} .
$$

The mixed-layer depth, entrainment rate and potential temperature jump were determined graphically, with the jump the difference between the extremes just above and below the mixed-layer top. The column just after $\Delta \theta$ is a prediction of it, described by (4.1). $F_{\mathrm{i}}$ and $B_{0}$ are the same as the thermal energy fluxes in the run labels (units of W $\mathrm{m}^{-2}$ ) converted into buoyancy fluxes by multiplication by $g /\left(\rho c_{p} \theta\right) \approx 2.92 \times 10^{-5} \mathrm{~m}^{2} \mathrm{~kg}^{-1}$.

The last column of Table $\mathrm{I}$ is the negative of the r.h.s. of (1.6), and should therefore be the same as $B_{\mathrm{i}}$. Actually, its value is about $20 \%$ smaller, fairly uniformly. This indicates that Lilly's (2002b) estimate of $A_{n}=3$ is about $20 \%$ too small, if we assume that the higher resolution simulations used here are more accurate.

Figures 1-5 show important time- or height-dependent aspects of five of the simulations, 0030, 3000, 6000, $60 \mathrm{~m} 10$ and $60 \mathrm{~m} 20$. These include the temporal growth of the mixed-layer depth, equivalent to the entrainment rate, and height profiles of the mean potential temperature, the mean kinetic energy to the $3 / 2$ power, the dissipation rate, and their ratio, $e^{3 / 2} / \varepsilon$, a turbulent length scale, all averaged over the last 13000 seconds.

The entrainment rate shows quasi-periodic fluctuations for all runs. These are found to be essentially spurious,

Table I. Results of smoke cloud simulations.

\begin{tabular}{|c|c|c|c|c|c|c|c|c|}
\hline Run & $\begin{array}{l}z_{\mathrm{i}} \\
\mathrm{m}\end{array}$ & $\begin{array}{c}w_{\mathrm{e}} \\
10^{-2} \mathrm{~m} \mathrm{~s}^{-1}\end{array}$ & $\begin{array}{c}\Delta \theta \\
\mathrm{K}\end{array}$ & $\begin{array}{l}\Delta \theta_{\text {pred }} \\
\mathrm{K}\end{array}$ & $\begin{array}{c}B_{\mathrm{i}} \\
10^{-4} \mathrm{~m}^{2} \mathrm{~s}^{-3}\end{array}$ & $\begin{array}{c}F_{\mathrm{i}} \\
10^{-4} \mathrm{~m}^{2} \mathrm{~s}^{-3}\end{array}$ & $\begin{array}{c}B_{0} \\
10^{-4} \mathrm{~m}^{2} \mathrm{~s}^{-3}\end{array}$ & $\frac{B_{0}}{4}+\frac{F_{\mathrm{i}}}{2}$ \\
\hline 0030 & 708 & 0.125 & 6.3 & 6.2 & -2.67 & 0 & 8.77 & 2.19 \\
\hline 0060 & 715 & 0.250 & 5.9 & 5.9 & -4.98 & 0 & 17.54 & 4.38 \\
\hline 1545 & 722 & 0.306 & 6.1 & 6.1 & -6.32 & 4.38 & 13.14 & 5.48 \\
\hline 3000 & 715 & 0.198 & 7.3 & 6.6 & -4.90 & 8.77 & 0 & 4.38 \\
\hline 3030 & 725 & 0.354 & 6.25 & 6.3 & -7.47 & 8.77 & 8.77 & 6.58 \\
\hline 3060 & 735 & 0.583 & 5.9 & 6.0 & -11.62 & 8.77 & 17.54 & 8.77 \\
\hline 4530 & 728 & 0.472 & 6.7 & 6.35 & -10.69 & 13.14 & 8.77 & 8.77 \\
\hline $60 \mathrm{~m} 20$ & 728 & 0.333 & 7.6 & 6.9 & -8.55 & 17.54 & -5.85 & 7.31 \\
\hline $60 \mathrm{~m} 10$ & 728 & 0.340 & 7.6 & 6.8 & -8.72 & 17.54 & -2.92 & 8.04 \\
\hline 6000 & 728 & 0.389 & 7.4 & 6.7 & -9.73 & 17.54 & 0 & 8.77 \\
\hline 6030 & 744 & 0.625 & 6.7 & 6.4 & -14.16 & 17.54 & 8.77 & 10.96 \\
\hline 6060 & 755 & 0.806 & 6.1 & 6.1 & -16.59 & 17.54 & 17.54 & 13.16 \\
\hline Av & 727 & 0.398 & 6.65 & 6.36 & -8.87 & 11.96 & 8.37 & 7.40 \\
\hline
\end{tabular}



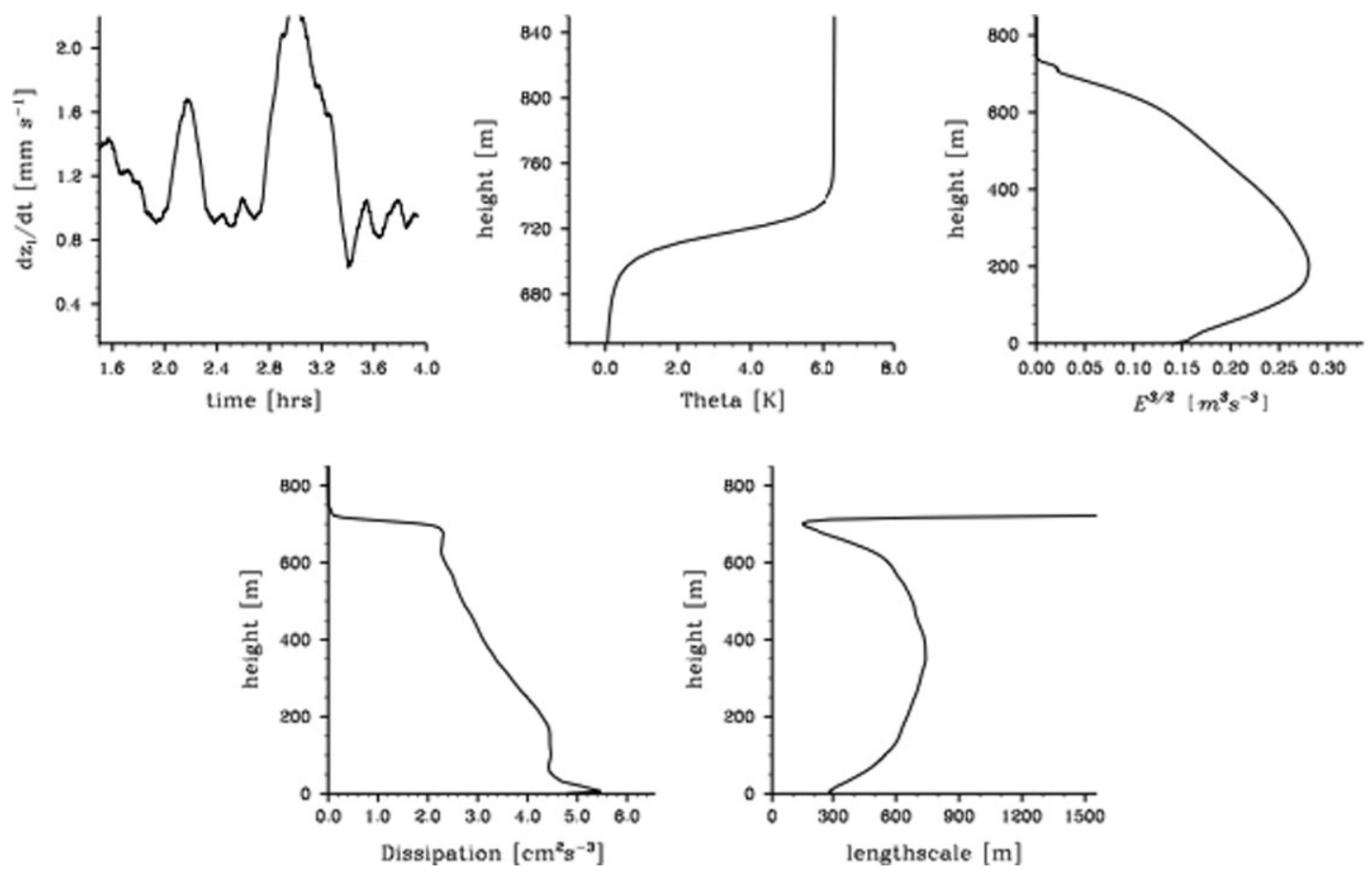

Figure 1. Statistics for run 0030, with no radiative cooling and surface heating of $30 \mathrm{~W} \mathrm{~m}^{-2}$ : (a) time record of mixed-layer growth due to entrainment; (b) height profile of potential temperature deviation from the mixed-layer value; (c) height profile of the $3 / 2$ power of kinetic energy; (d) height profile of the energy dissipation rate; (e) height profile of the energy length scale, the ratio of the plots on (c) and (d).
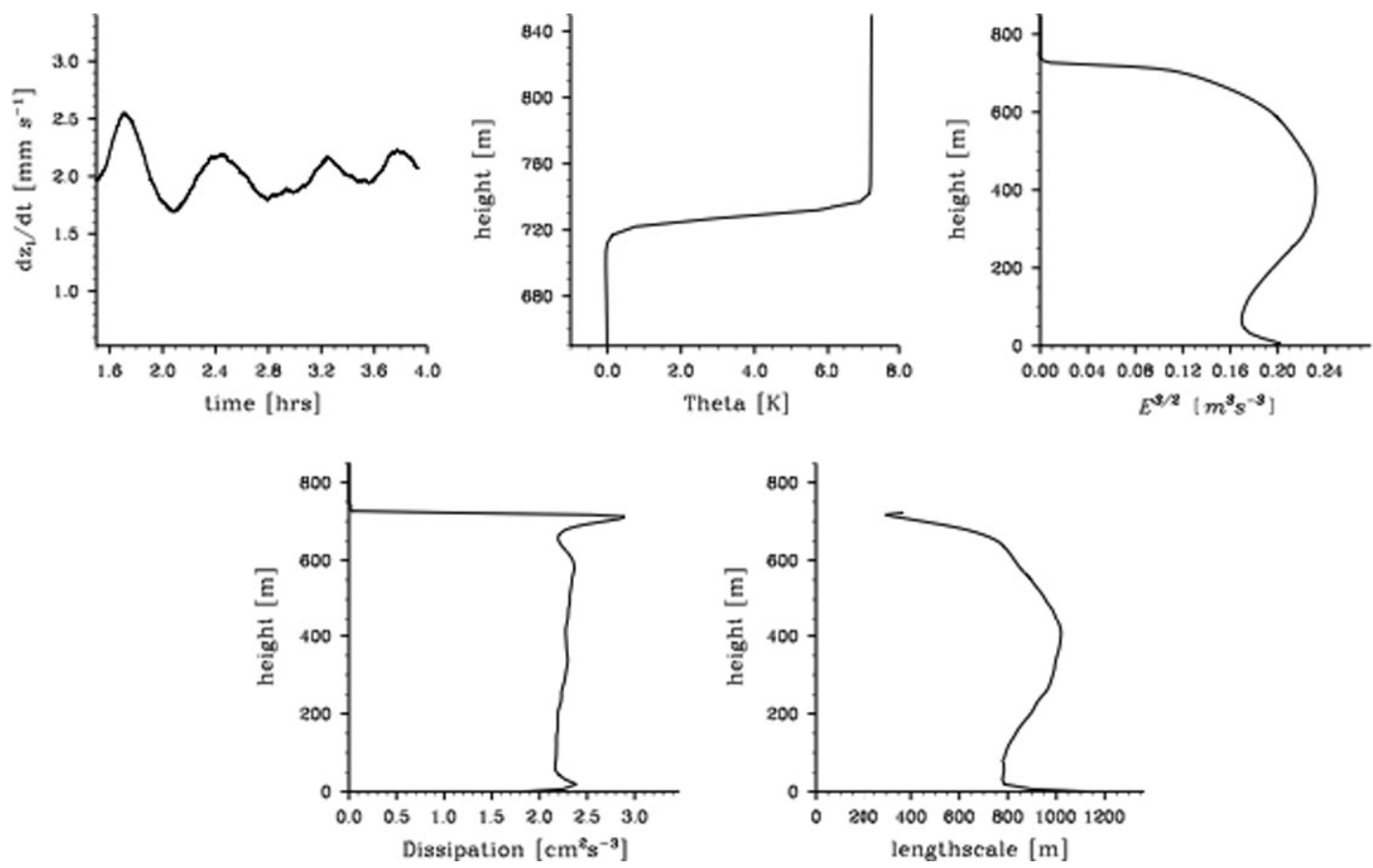

Figure 2. Same as Figure 1 but for run 3000.

caused by the mixed-layer top moving across grid levels periodically.

The potential temperature profiles are used to determine $\Delta \theta$. Their smooth appearance at the mixed-layer top is mainly due to spatial and temporal fluctuations of the mixed-layer height.

The profiles of $e^{3 / 2}$ are all fairly similar in shape, but the maxima are higher when the buoyancy driving is all or mainly radiative. The dissipation profiles are mostly linear, but again maximized near the level of principal driving. The ratio of these, the length scale, generally shows a maximum in the middle and minima near both boundaries, as might be intuitively expected. This is not true, however, for 6000, nor for 6030, 6060 and 4530, for each of which the length scale is nearly constant or weakly sloped with height. 

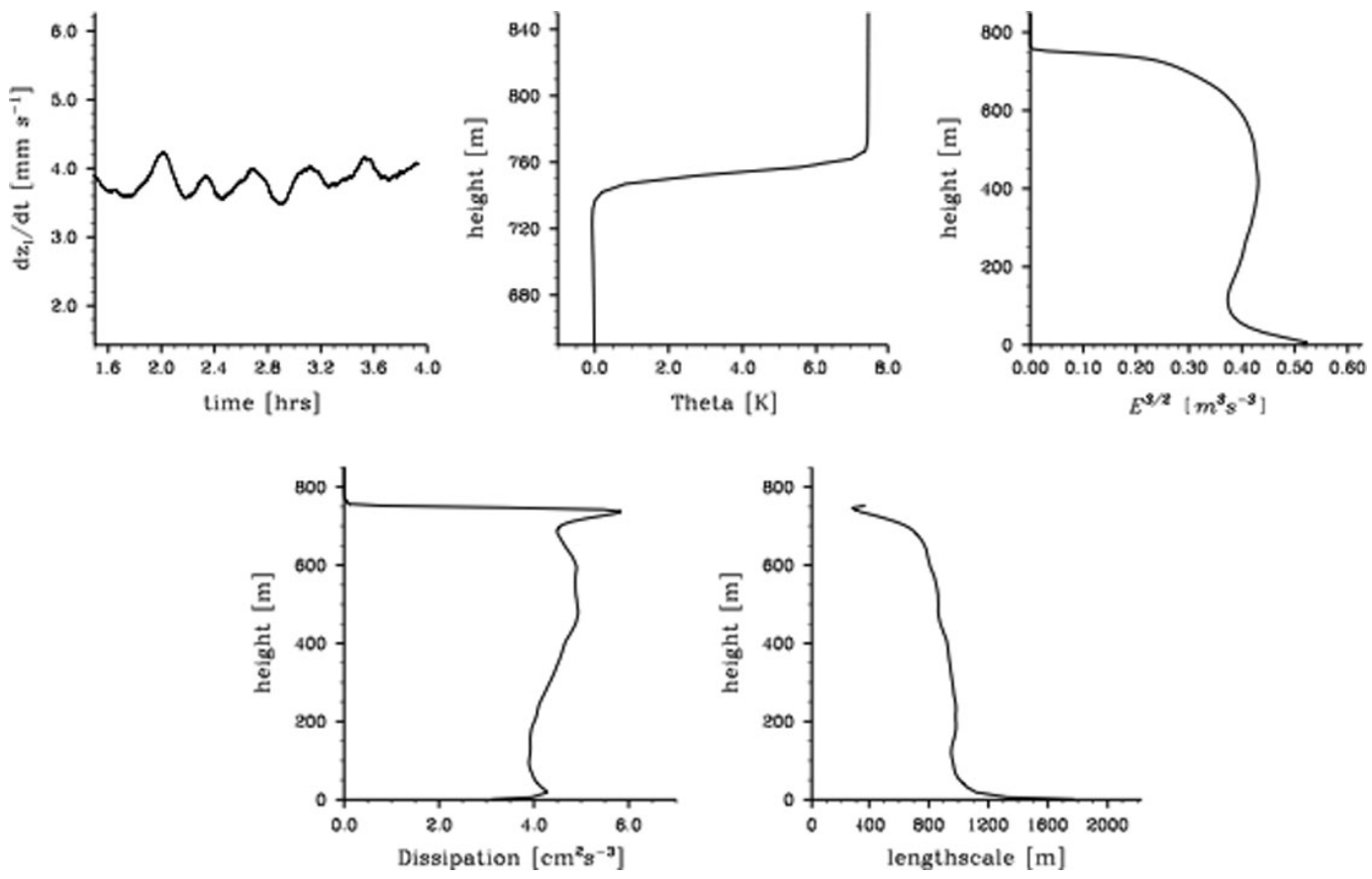

Figure 3. Same as Figure 1 but for run 6000.
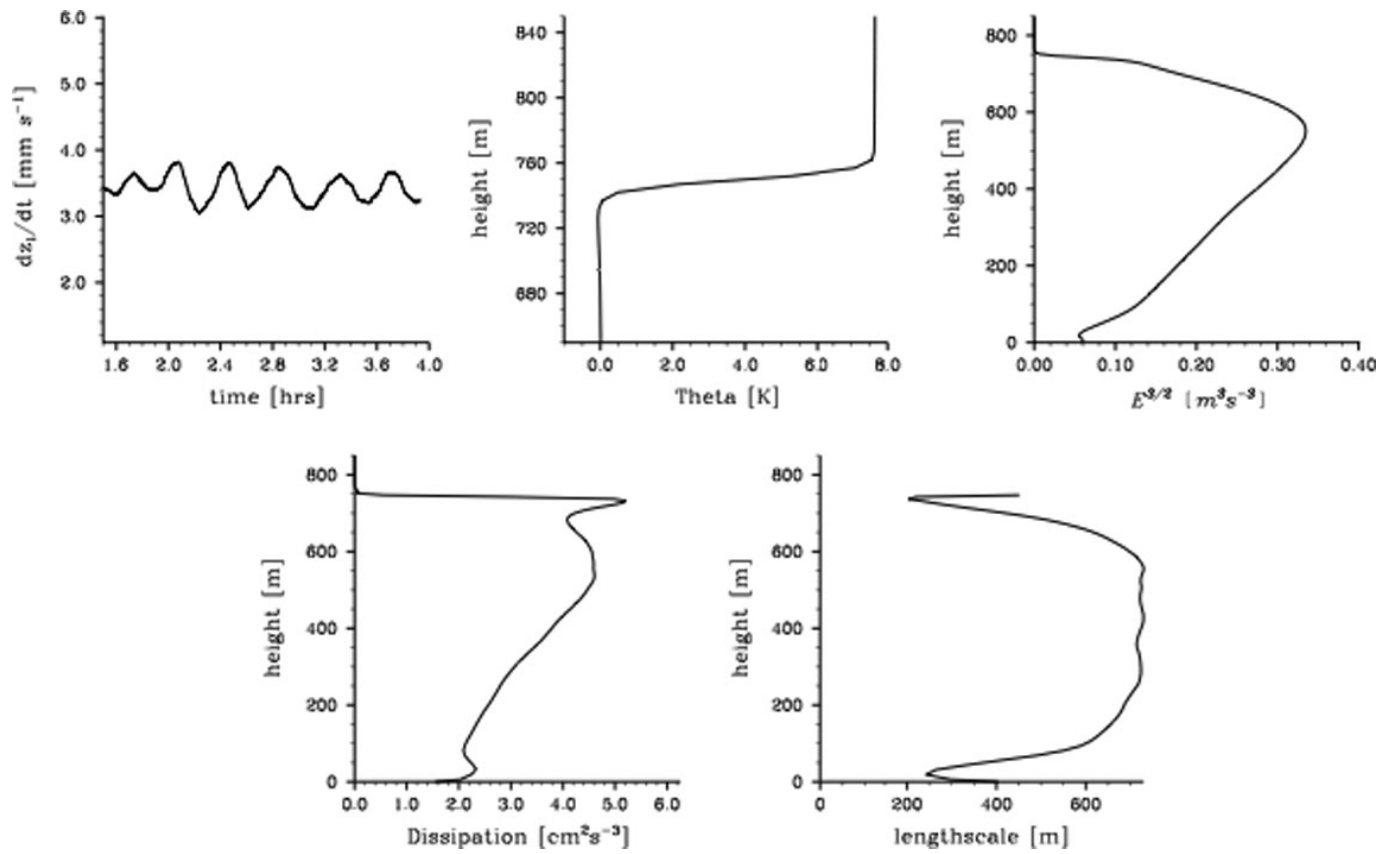

Figure 4. Same as Figure 1 but for run $60 \mathrm{~m} 10$, with surface cooling of $10 \mathrm{~W} \mathrm{~m}^{-2}$.

The two simulations forced by negative buoyancy flux at the surface were carried out to test the predictions in sections 1 and 3 of the greatest negative surface flux that allows maintenance of turbulence to the surface. As shown by Figures 4 and 5, in both runs there is a change in slope of the energy and dissipation rate near the surface, but only for $60 \mathrm{~m} 20$ do they both nearly vanish. Although not shown in these plots, for $60 \mathrm{~m} 20$ the buoyancy flux gradient is strongly positive in the lowest $40 \mathrm{~m}$, indicating strong subgrid-scale diffusion, a feature almost absent in $60 \mathrm{~m} 10$. These results are not very clear-cut, but suggest that $60 \mathrm{~m} 10$ is near the decoupling limit, while $60 \mathrm{~m} 20$ is evidently beyond it. The $1 / 6^{\text {th }}$ ratio of $B_{i 0} / B_{i}$ for $60 \mathrm{~m} 10$ is close to the $2 / 15^{\text {ths }}$ prediction of (1.11) and (1.13).

\section{Energy equation solution}

Since the flux closure was derived with implicit reference to the kinetic energy balance, a solution of the energy equation might allow a better test of its validity. We show one relatively simple solution here, and draw some 

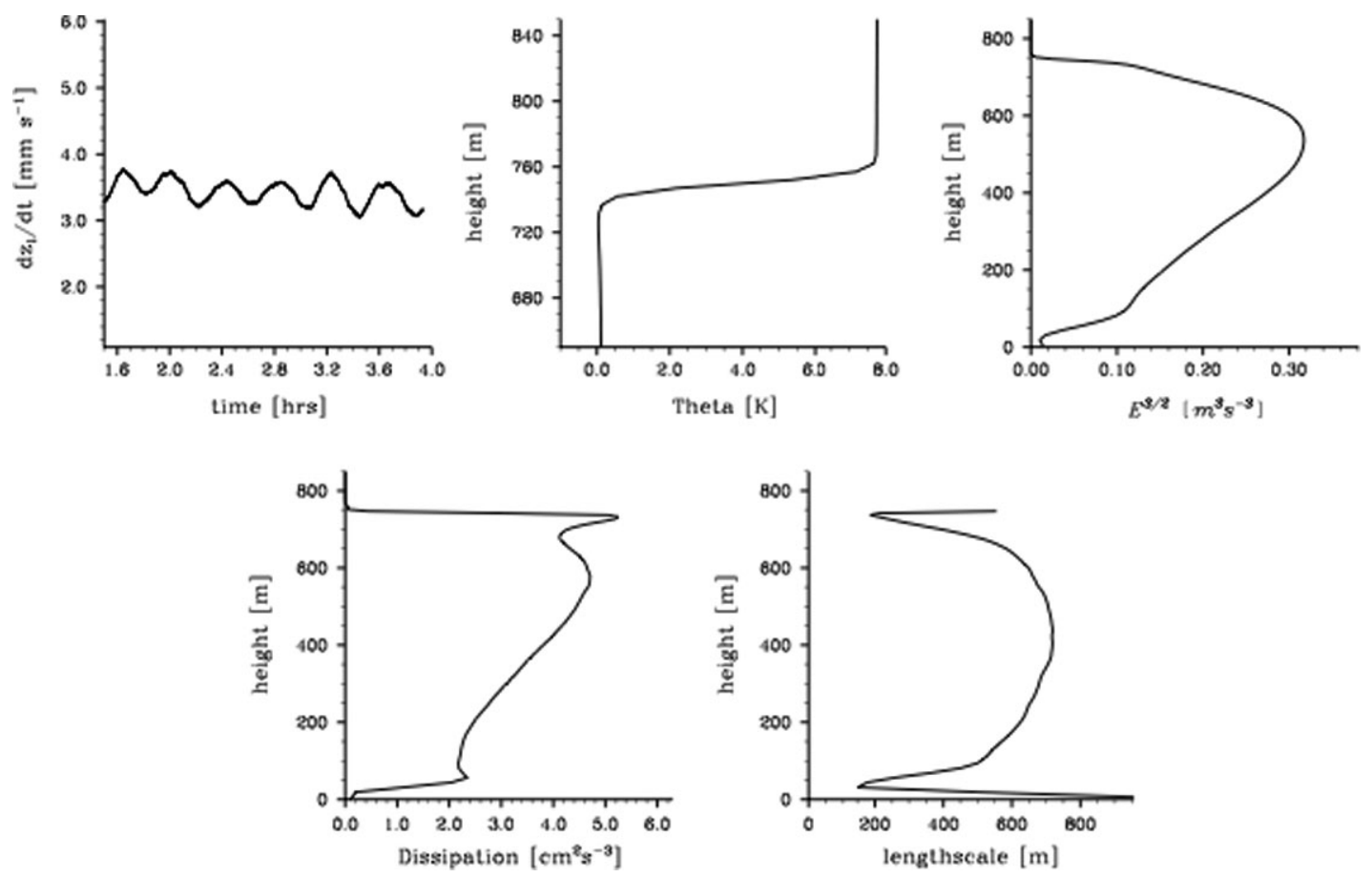

Figure 5. Same as Figure 1 but for run $60 \mathrm{~m} 20$, with surface cooling of $20 \mathrm{~W} \mathrm{~m}^{-2}$.

conclusions from it. A problem with such solutions is that they depend on several somewhat arbitrary functions and constants. Here the three or four arbitrary constants are determined backwards, so that the solutions are forced to match observations and simulations. This seems appropriate to the present purpose, which is to determine whether the flux closure is reasonably consistent with turbulence theory.

A symbolic form of the turbulent kinetic energy equation is

$$
\frac{\partial e}{\partial t}=G e n+D i f f-\varepsilon,
$$

where Gen stands for generation terms, typically from shear and buoyancy, Diff stands for the diffusion of kinetic energy and the gradient of the work done against pressure force, and $\varepsilon$ is the viscous dissipation. Here we approximate these terms in conventional ways. We ignore the time derivative, on the usual grounds that it is generally much smaller than the r.h.s. components. For generation, we apply only buoyancy flux $B$, given by the linear expressions of (1.3):

$$
\text { Gen }=B=B_{0}\left\{1-\left(z / z_{\mathrm{i}}\right)\right\}+\left(B_{\mathrm{i}}+F_{\mathrm{i}}\right) z / z_{\mathrm{i}} \text { for } z<z_{\mathrm{i}} .
$$

Diff is approximated as a turbulent diffusion of kinetic energy, that is,

$$
D i f f=\frac{\partial}{\partial z}\left(c \ell e^{1 / 2} \frac{\partial e}{\partial z}\right),
$$

where $c \ell e^{1 / 2}$ is an eddy diffusivity, with $c$ a dimensionless constant and $\ell$ a length scale, to be specified.
Dissipation is approximated in a typical form, assuming an inertial subrange transferring energy downscale, as

$$
\varepsilon=c_{\varepsilon} e^{3 / 2} / \ell
$$

where $c_{\varepsilon}$ is another dimensionless constant and $\ell$ is assumed to be the same length scale.

The present analysis is done using a uniform length scale

$$
\ell=z_{\mathrm{i}}
$$

While this is not an accurate assumption, as shown by the above-discussed profiles of $e^{3 / 2} / \varepsilon$, the general character of the solution is evidently not strongly dependent on that inaccuracy. A solution using a more realistic length-scale assumption has been developed by the first author, and may be presented elsewhere. With the present assumption, the energy equation may be written in a form suitable for solution as

$$
\begin{aligned}
& \frac{\mathrm{d}^{2} e^{3 / 2}}{\mathrm{~d}\left(z / z_{\mathrm{i}}\right)^{2}}-\alpha^{2} e^{3 / 2}=-\frac{3}{2} \frac{z_{\mathrm{i}}}{c} B \\
& =-\frac{\alpha^{2} z_{\mathrm{i}}}{c_{\varepsilon}}\left\{B_{0}-\left(B_{0}-B_{\mathrm{i}}-F_{\mathrm{i}}\right) \frac{z}{z_{\mathrm{i}}}\right\},
\end{aligned}
$$

where $\alpha$ is a dimensionless wave number, given by

$$
\alpha^{2}=\frac{3}{2} \frac{c_{\varepsilon}}{c} .
$$

The equations and analysis to this point are fairly similar to that of Zilitinkevich and Mironov (1992), which they applied to a prediction of the thermocline in 
a freshwater basin. The subsequent development differs considerably, however.

A trial solution to (3.6) may be written in the form

$$
\begin{aligned}
e^{3 / 2}= & c_{1}+c_{2} \frac{z}{z_{\mathrm{i}}}+c_{3} \cosh \left(\alpha \frac{z}{z_{\mathrm{i}}}\right) \\
& +c_{4} \cosh \left\{\alpha\left(1-\frac{z}{z_{\mathrm{i}}}\right)\right\},
\end{aligned}
$$

where $c_{1}$ and $c_{2}$ are obtained by direct substitution and $c_{3}$ and $c_{4}$ are chosen to fit boundary conditions. The first two are

$$
c_{1}=\frac{z_{\mathrm{i}} B_{0}}{c_{\varepsilon}}, c_{2}=-\frac{z_{\mathrm{i}}}{c_{\varepsilon}}\left(B_{0}-B_{\mathrm{i}}-F_{\mathrm{i}}\right) .
$$

We choose boundary conditions on the same basis as Zilitinkevich and Mironov (1992), who assume vanishing energy flux, that is $\mathrm{d} e^{3 / 2} / \mathrm{d} z=0$, at both boundaries. This ignores possible export of energy by gravity waves and insures that the vertical integrals of generation and dissipation are equal. The vertical derivative of (3.8) is

$$
\begin{aligned}
& \frac{\mathrm{d} e^{3 / 2}}{\mathrm{~d}\left(z / z_{\mathrm{i}}\right)}=c_{2}+\alpha c_{3} \sinh \left(\alpha \frac{z}{z_{\mathrm{i}}}\right) \\
& -\alpha c_{4} \sinh \left\{\alpha\left(1-\frac{z}{z_{\mathrm{i}}}\right)\right\}=0 \text { at } z=0, z_{\mathrm{i}}
\end{aligned}
$$

Thus, the two last coefficients are given by

$$
c_{3}=-\frac{c_{2}}{\alpha \sinh \alpha}=-c_{4}
$$

The solution of (3.6) is then

$$
\begin{aligned}
e^{3 / 2} & =\frac{z_{\mathrm{i}}}{c_{\varepsilon}}\left\{B_{0}+\left(B_{0}-B_{\mathrm{i}}-F_{\mathrm{i}}\right)\right. \\
& \left.\left(-\frac{z}{z_{\mathrm{i}}}+\frac{\cosh \left(\alpha z / z_{\mathrm{i}}\right)-\cosh \left\{\alpha\left(1-z / z_{\mathrm{i}}\right)\right\}}{\alpha \sinh \alpha}\right)\right\} .
\end{aligned}
$$

The dissipation rate, from (3.4), is the quantity in large curly brackets,

$$
\begin{aligned}
& \varepsilon=B_{0}+\left(B_{0}-B_{\mathrm{i}}-F_{\mathrm{i}}\right) \\
& \left(-\frac{z}{z_{\mathrm{i}}}+\frac{\cosh \left(\alpha z / z_{\mathrm{i}}\right)-\cosh \left\{\alpha\left(1-z / z_{\mathrm{i}}\right)\right\}}{\alpha \sinh \alpha}\right)
\end{aligned}
$$

At the boundary $z=z_{\mathrm{i}}$, this is

$$
\left.\begin{array}{c}
\varepsilon_{\mathrm{i}}=B_{\mathrm{i}}+F_{\mathrm{i}}+\left(B_{0}-B_{\mathrm{i}}-F_{\mathrm{i}}\right) \frac{\cosh \alpha-1}{\alpha \sinh \alpha} \\
=B_{\mathrm{i}}+F_{\mathrm{i}}+\left(B_{0}-B_{\mathrm{i}}-F_{\mathrm{i}}\right) T \\
\text { where } T=\frac{\cosh \alpha-1}{\alpha \sinh \alpha} \equiv \frac{1}{\alpha} \tanh \frac{\alpha}{2}
\end{array}\right\} .
$$

We now define the ratio of the buoyancy flux to dissipation at the boundary, i.e.

$$
c_{\mathrm{i}}=-B_{\mathrm{i}} / \varepsilon_{\mathrm{i}},
$$

and then combine terms of (3.14) to yield

$$
B_{0} T+B_{\mathrm{i}}\left(1-T+\frac{1}{c_{\mathrm{i}}}\right)+F_{\mathrm{i}}(1-T)=0 .
$$

It is now evident that, with $T$ and $c_{\mathrm{i}}$ constants, (3.16) is equivalent to (1.6), and therefore that the energy equation yields similar solutions to those of the flux closure. Actually, the solutions of the energy equation seem almost irrelevant, since they are deduced after explicitly choosing the flux profiles. The value of the energy equation, however, comes from comparison of its coefficients with those of the flux profiles. After eliminating $B_{i}$ from (1.6) and (3.16), the coefficients of $B_{0}$ and $F_{\mathrm{i}}$ must vanish, yielding

$$
T=\frac{1}{n+2} \approx 1 / 3, c_{\mathrm{i}}=\frac{A_{n}}{n+1} \approx \frac{3}{2} .
$$

Numerical solution of the second of (3.14) then yields

$$
\alpha \approx 2.576 \text {, and therefore } c_{\varepsilon} / c=2 \alpha^{2} / 3 \approx 4.424 \text {. }
$$

Thus the height-weighting exponent $n$ is dependent on the ratio of the energy dissipation and diffusion coefficients (or vice versa). By direct evaluation one can show that if that coefficient ratio, $c_{\varepsilon} / c$, is reduced by half, indicating stronger diffusive energy transfer, then $n$ is also reduced by about half, and if $c_{\varepsilon} / c$ is doubled, $n$ is about doubled. Stronger diffusion reduces the loss of surface-generated energy by dissipation and weaker diffusion increases it. Vanishing $n$, that is the more conventional flux closure assumption, implies vanishing dissipation. The height-weighting assumption in (1.1) was assumed by Lilly (2002b) as an empirical fix. The above results probably do not prove (1.1) to be the only or best closure assumption, but they do seem to show that, within its constraints, an optimal $n$ is directly related to the parameters of the kinetic energy (KE) equation, and these, along with the forcing fluxes, determine the KE solution.

We now compare the nominal constants $c_{\mathrm{i}}$ and $T$ with the simulation results. The first of these, $c_{\mathrm{i}}$, is obtained from (3.15) as the ratio of $-B_{\mathrm{i}}$ and $\varepsilon_{\mathrm{i}}$. Individual values, which range from 1.24 to 2.36 are not shown, but the average value for the 12 cases is 1.87 , compared to the analytic value 1.5 . The values of $T$ are obtained by evaluating (3.13) at $z=0$ and $z_{\mathrm{i}}$, for which the fraction in brackets is either positive or negative $T$. Specifically, we evaluate

$$
2 T=1+\frac{\varepsilon_{\mathrm{i}}-\varepsilon_{0}}{B_{0}-B_{\mathrm{i}}-F_{\mathrm{i}}}
$$

Again, we do not display the individual values of $T$, which range from 0.34 to 0.46 . The average is 0.378 , as compared to the analytic prediction of $1 / 3$. Thus $c_{\mathrm{i}}$ and $T$ each differ from predictions by $15-25 \%$.

The simulations also provide data suitable for evaluating the energy balance of the mixed layer, as given by the 
terms in (3.6). The height integral of the generation and dissipation terms should be equal if the buoyancy fluxes do indeed vanish at the boundary, but this seems not to be true by a few percent for some of the runs. As is typically the case, kinetic energy is diffused from the regions where it is most strongly generated to those where it is weakly generated or removed. The removal by entrainment does not directly show up, due to the assumed vanishing depth of the radiating region, but $B_{\mathrm{i}} z / z_{\mathrm{i}}$ contributes negatively to the balance at all levels.

The case of a mixed layer cooled radiatively at the top and by negative surface flux at the bottom can also be considered with the aid of the energy equation. Here, the approach is to assume that $e$ vanishes at $z=0$, so that the surface is marginally decoupled from turbulence generated at the top. From (3.8), this requires that

$$
c_{1}+c_{3}+c_{4} \cosh \alpha=0 \text {. }
$$

After substitution of (3.9), (3.11) and (3.17) this leads to the condition

$$
2 B_{0}+B_{\mathrm{i}}+F_{\mathrm{i}}=0 .
$$

When combined with the upper boundary condition, that is

$$
B_{\mathrm{i}}=-B_{0} / 4-F_{\mathrm{i}} / 2,
$$

$B_{i}$ may be eliminated, leaving the surface and entrainment fluxes:

$$
B_{0}=-\frac{F_{\mathrm{i}}}{2 / T-5 / 2}=-\frac{2 F_{\mathrm{i}}}{7}, \quad B_{\mathrm{i}}=-\frac{3 F_{\mathrm{i}}}{7} .
$$

These are not directly dependent on $\alpha$. The first is more than twice that shown in (1.9) from the modified flux closure model, for reasons that are not obvious to us. The simulation results suggest that the decoupling limit of $-B_{0} / F_{\mathrm{i}}$ is probably smaller than $1 / 4^{\text {th }}$, perhaps near $1 / 6^{\text {th }}$. The relative merits of the flux closure and energy equation closure are not clear-cut.

\section{Tests of simulation quality}

Although the theory and the simulations agree in important respects, they also disagree in some. In this section we examine some of these disagreements, and make some further tests on the validity of the simulations.

In Table I, three runs, 3060, 6030 and 6060, have unusually high values of entrainment flux compared to that predicted by the last column. A possible reason for the high values is an amplifying interaction between energy generated by surface and by radiative forcing, so that the linear assumption (1.6) is incorrect for combined forcing. However, three other runs with combined positive forcing, 1545, 3030 and 4530, exhibit smaller entrainment rates.

An interesting and moderately important statistic is the dissipative length scale, $e^{3 / 2} / \varepsilon$, which by (3.4) is $\ell / c_{\varepsilon}$.
As shown above, most runs have a profile peaked in the middle and nearly symmetric with height, while the 6000 run is flattened in the middle and quite asymmetric. Also, for 4530, 6030 and 6060 (none shown here) the maximum is weak and near the upper boundary. This anomalistic behaviour for runs with large radiative forcing is mainly associated with similar anomalies of the energy, since the dissipation rates are nearly uniform with height for all these runs. Interestingly, the runs with negative surface forcing, $60 \mathrm{~m} 10$ and $60 \mathrm{~m} 20$, show much more 'regular' length-scale profiles. These results suggest a possible defect in the simulations, especially near the boundaries.

To put a more rigorous test on the simulations, we worked out an analytic solution for comparison with the results, again with similarities to Zilitinkevich and Mironov (1992). We start with the heat balance equation

$$
\frac{\partial \bar{\theta}}{\partial t}=-\frac{\partial}{\partial z}\left(\overline{w^{\prime} \theta^{\prime}}+F\right)=\frac{\left(B_{0}-B_{\mathrm{i}}-F_{\mathrm{i}}\right) \bar{\theta}}{g z_{\mathrm{i}}},
$$

and the basic entrainment condition, that is

$$
w_{e}=\frac{\mathrm{d} z_{\mathrm{i}}}{\mathrm{d} t}=-\frac{\bar{\theta} B_{\mathrm{i}}}{g \Delta \theta}=-\frac{\bar{\theta} B_{\mathrm{i}}}{g\left(\theta_{\mathrm{i}}-\bar{\theta}\right)},
$$

where $\theta_{i}$ is the upper environmental temperature. By dividing (4.1) by (4.2), we obtain a relation between the changes in $\theta$ and $z_{\mathrm{i}}$, that is

$$
\frac{\mathrm{d} \bar{\theta}}{\mathrm{d} z_{\mathrm{i}}}=-\frac{c_{p} \bar{\theta}}{g} \frac{B_{0}-B_{\mathrm{i}}-F_{\mathrm{i}}}{B_{\mathrm{i}}} \frac{\theta_{\mathrm{i}}-\bar{\theta}}{z_{\mathrm{i}}} .
$$

The first fraction is, supposedly, a function of the forcing only, so that, after using (1.6) and (1.12), we obtain

$$
\frac{\mathrm{d} \bar{\theta}}{\mathrm{d} z_{\mathrm{i}}}=\frac{3}{2} \frac{3 B_{0}-F_{\mathrm{i}}}{B_{0}+2 F_{\mathrm{i}}} \frac{\theta_{i}-\bar{\theta}}{z_{\mathrm{i}}} .
$$

The simplest result is obtained by neglecting the small increase with height of $\theta_{\mathrm{i}}$, which changes the result insignificantly. Integration of (4.4) then yields

$$
\frac{\theta_{\mathrm{i}}-\bar{\theta}}{\theta_{\mathrm{i}}-\bar{\theta}_{0}}=\left(\frac{z_{\mathrm{i}}}{z_{\mathrm{i} 0}}\right)^{-n} \text {, where } n=\frac{3}{2} \frac{3 B_{0}-F_{\mathrm{i}}}{B_{0}+2 F_{\mathrm{i}}} \text {. }
$$

We now use (4.6) to replace the denominator in (4.3), which allows the latter to be integrated in time to obtain

$$
\left(\frac{z_{\mathrm{i}}}{z_{\mathrm{i} 0}}\right)^{1-n}=1+\frac{-B_{0}+F_{\mathrm{i}}}{z_{\mathrm{i} 0} \Delta \theta} t
$$

where $\Delta \theta$ is taken to be the initial value $7 \mathrm{~K}$. Although $1-n$ can be of either sign, $z_{\mathrm{i}}$ will always increase with time. After putting $B_{0}$ and $F_{\mathrm{i}}$ into the correct units $(\mathrm{K}$ $\mathrm{m} \mathrm{s}^{-1}$ ) and setting $t=6500 \mathrm{~s},(4.6)$ is easily evaluated, and we obtain the following results for all the runs except $60 \mathrm{~m} 10$, which is essentially midway between $60 \mathrm{~m} 20$ and 6000 . The last row contains the results of the simulation at $t=8000 \mathrm{~s}$, that is, about $6500 \mathrm{~s}$ after the mixed length started to grow. 
Table II. Predicted and simulated mixed layer heights.

\begin{tabular}{lccccccccccc}
\hline Run & 0030 & 0060 & 1545 & 3000 & 3030 & 3060 & 4530 & $60 \mathrm{~m} 20$ & 6000 & 6030 & 6060 \\
\hline$n$ & 4.50 & 4.50 & 2.40 & -0.75 & 1.00 & 1.88 & .562 & -1.80 & -.75 & .30 & 1.00 \\
Pre & 708 & 716 & 719 & 715 & 723 & 731 & 731 & 724 & 729 & 737 & 746 \\
Sim & 708 & 716 & 722 & 715 & 725 & 735 & 735 & 728 & 728 & 744 & 755 \\
\hline
\end{tabular}

We see that the height increases are generally well predicted, although usually a little smaller than the simulated values, especially for the last two runs. If the predicted or simulated value of $\Delta \theta$, from Table I, were used, the predicted height would usually be too large. On the whole, this test seems to confirm the general validity of both the theory and the simulations, although the above apparent discrepancies remain unexplained.

Finally, we note that the entrainment fluxes for surface driving, cases 0030 and 0060 , are almost $50 \%$ greater than those usually obtained by direct flux measurements from observations or simulations. This is, we believe, because the commonly observed maximum negative flux is reduced by averaging of spatial and temporal fluctuations of the mixed-layer top, as proposed by Lilly (2002a,b).

\section{Conclusions}

The test of the Lilly (2002a) entrainment flux closure algorithm against the analytic solution of the energy equation appears to be successful, in that its special feature, the height weighting of the buoyancy flux integral, is found to be uniquely related to the ratio of the amplitude coefficients for dissipation and diffusion, which is also the principle parameter determining solutions of the turbulent kinetic energy equation. Solutions of the energy equation found here are somewhat limited, because of their assumption of a constant scale height, but their dissipation rates agree fairly well with those of large-eddy simulations. The simulations, though carried out with greater resolution than most previously shown results, show some unexplained anomalies in the dissipation length scale, but agree well with the theory in their prediction of mixed-layer growth by entrainment. A side issue investigated here is the question of the maximum negative buoyancy flux that allows turbulence generated by cloud-top radiative cooling to extend to the surface. For this situation, predictions generated by the flux closure and the energy equation differ significantly, but the decoupling criteria are not sharply enough defined to allow a clear choice.

\section{Acknowledgements}

In addition to the acknowledgements listed in II, we have accepted and acknowledge the recommendation by an anonymous reviewer to alter the title wording from "Verification" to "Validation".

\section{References}

Bretherton CS, MacVean MK, Bechtold P, Chlond A, Cotton WR, Cuxart J, Cuijpers JWM, Khairoutdinov M, Kosovic B, Lewellen D, Moeng C-H, Siebesma AP, Stevens B, Stevens DE, Sykes I, Wyant MC. 1997. An intercomparison of radiatively driven entrainment and turbulence in a smoke cloud, as simulated by different numerical models. Q. J. R. Meteorol. Soc. 125: 391-423.

Lilly DK. 1968. Models of cloud-topped mixed layers under a strong inversion. Q. J. R. Meteorol. Soc. 94: 292-309.

Lilly DK. 2002a. Entrainment into mixed layers. Part I: Sharp-edged and smoothed tops. J. Atmos. Sci. 59: 3340-3352.

Lilly DK. 2002b. Entrainment into mixed layers. Part II: A new closure. J. Atmos. Sci. 59: 3353-3361.

Stevens B, Moeng C-H, Ackerman AS, Bretherton CS, Chlond A, de Roode S, Edwards J, Golaz J-C, Jiang H, Khairoutdinov M, Kirkpatrick MP, Lewellen DC, Lock A, Müller F, Stevens DE, Whelan E, Zhu P. 2005. Evaluation of large-eddy simulations via observations of nocturnal marine stratocumulus. Mon. Weather Rev. 133: $1443-1462$

Zilitinkevich SS, Mironov DV. 1992. Theoretical model of the thermocline in a freshwater basin. J. Phys. Oceanogr. 22: 988-996. 